\title{
Application of the Golden Numerical Rings for Configure Acoustic Systems of Fine Resolution
}

\author{
W. RIZNYK ${ }^{a, b, *}$ \\ ${ }^{a}$ University of Technology and Life Sciences of Bydgoszcz, al. S. Kaliskiego 7, 85-796 Bydgoszcz, Poland \\ ${ }^{b}$ Lviv Polytechnic National University, Ukraine
}

The paper presents a new mathematical principle for improving the quality indices of acoustic systems with non-uniform structure (e.g. overlapping masks utilizing the entire ultra-acoustic aperture) with respect to the wavelength scale, where each pair of holes serves as an interferometer selecting the signal component at a spatial frequency, using novel design based on the combinatorial sequencing theory, namely the concept of golden numerical rings. The concept can be used for finding optimal placement of structural elements in spatially distributed systems, for example, positioning of holes elements in sonar antenna array. Golden numerical rings are cyclic sequences of positive integers which form perfect partitions of a finite interval $[1, S]$ of integers. The sums of connected sub-sequences of a GRB enumerate the set of integer $[1, S]$ exactly $R$-times. These design techniques make it possible to configure non-redundant masks in order to avoid interference of signal components of the same spatial frequency because phases of the components are unknown.

PACS: 43.28.Js

\section{Introduction}

\subsection{Problem for constructing non-redundant arrays}

General problem of optimization of acoustic systems relates to finding the best placement of its structural elements in spatially distributed systems as well as a better understanding of the role of geometric structure in the behavior of natural and man-made objects. Research into underlying mathematical area involves the appropriate algebraic constructions based on finite groups in extensions of the Galois fields [1]. Application of the algebraic constructions and modern combinatorial analysis provides optimal solution of a lot of problems of high-resolution interferometry for radar, data communications, and signal design [2]. Some regular methods for constructing non-redundant two-dimensional $n$-element masks over $n \times n$ grids, based on algebraic combinatorial configurations such as difference sets are suggested in the publication [3]. However, the classical theory of combinatorial configurations can hardly be expected effective for constructing high-resolution $2 \mathrm{D}$ or $3 \mathrm{D}$ acoustic systems, and finding optimal solutions for other problems in constructing microwave-, ultrasound or hydro-acoustic systems including low side lobe design. Hence, both an advanced theory and regular method for optimal solution of the problems are needed. In this paper is suggested a regular method for constructing linear, planar or 3D non-redundant arrays of acoustic systems with an arbitrarily large number of elements (openings or sensors),

\footnotetext{
* e-mail: wriz@utp.edu.pl
}

and systems of non-overlapping masks utilizing the entire aperture, based on similar one-dimensional problem solution, namely the concept of golden numerical rings (GNRs).

\section{Concept of the golden numerical rings}

\subsection{Perfect number sequence of daisy-chain topology}

Let us calculate all sums of the terms in the numerical $n$-element daisy-chain sequence of distinct positive integers $K_{n}=\left\{k_{1}, k_{2}, \ldots, k_{n}\right\}$, where we require all terms in each sum to be consecutive elements of the sequence. Easy to see the maximum of such sum is the sum $k_{1}+k_{2}+\ldots+k_{n}=S_{\max }^{\prime}$ of all $n$ elements, and the maximum number of distinct sums is

$$
S_{\max }^{\prime}=n(n+1) / 2 \text {. }
$$

The perfect number sequence is such $n$-element of daisy-chain topology $K_{n}=\left\{k_{1}, k_{2}, \ldots k_{n}\right\}$, where set of all terms in each sum to be consecutive elements of the sequence, exhausts the natural row. Example: the number sequence $\{1,3,2\}$ containing three $(n=3)$ elements allows an enumeration of all numbers $1,2,3,4=1+3$, $5=3+2,6=1+3+2$, where $S_{\max }^{\prime}=n(n+1) / 2=6$, and each sum from 1 to 6 occurs exactly once. So, the $3-$ element daisy-chain sequence is perfect number sequence.

\subsection{Golden numerical rings}

An $n$-element sequence $K_{n}=\left\{k_{1}, k_{2}, \ldots, k_{i}, \ldots k_{n}\right\}$ as being cyclic, so that element $k_{n}$ is followed by $k_{1}$, is called a "ring sequence" [4] (Fig. 1).

Table of sums of consecutive terms in ring sequence $K_{n}=\left\{k_{1}, k_{2}, \ldots, k_{i}, \ldots k_{n}\right\}$ is given below (Table I). 


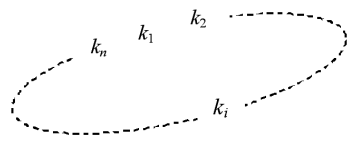

A sum of consecutive terms in the ring sequence is called "circular sum" [4]. The sum can have any of the $n$ terms as its starting point $p_{j}$, and finishing point $q_{j}$, and can be of any length (number of terms) from 1 to $n-1$.

Fig. 1. Ring sequence $\left\{k_{1}, k_{2}, \ldots, k_{i}, \ldots k_{n}\right\}$.

Sums of consecutive terms in ring sequence $K_{n}=\left\{k_{1}, k_{2}, \ldots, k_{i}, \ldots k_{n}\right\}$.

TABLE I

\begin{tabular}{c|c|c|c|c|c}
\hline \hline \multirow{2}{*}{$p_{j}$} & \multicolumn{5}{|c}{$q_{j}$} \\
\cline { 2 - 6 } & 1 & 2 & $\ldots$ & $n-1$ & $n$ \\
\hline 1 & $k_{1}$ & $\sum_{i=1}^{2} k_{i}$ & $\ldots$ & $\sum_{i=1}^{n-1} k_{i}$ & $\sum_{i=1}^{n} k_{i}$ \\
\hline 2 & $\sum_{i=1}^{n} k_{i}$ & $k_{2}$ & $\ldots$ & $\sum_{i=2}^{n-1} k_{i}$ & $\sum_{i=2}^{n} k_{i}$ \\
\hline$\ldots$ & & & $\ldots$ & & $\ldots$ \\
\hline$n-1$ & $\sum_{i=n-1}^{n} k_{i}+k_{1}$ & $\sum_{i=n-1}^{n} k_{i}+\sum_{i=1}^{2} k_{i}$ & $\ldots$ & $k_{n-1}$ & $\sum_{i=n-1}^{n} k_{i}$ \\
\hline$n$ & $k_{n}+k_{1}$ & $k_{n}+\sum_{i=1}^{2} k_{i}$ & $\ldots$ & $\sum_{i=1}^{n} k_{i}$ & $k_{n}$
\end{tabular}

So, each numerical pair $\left(p_{j}, q_{j}\right)$ corresponds to circular sum $S_{j}=S\left(p_{j}, q_{j}\right)$, and it can be calculated in case, when $p_{j} \leq q_{j}$, by equation

$$
S_{j}=S\left(p_{j}, q_{j}\right)=\sum_{i=p_{j}}^{q_{j}} k_{i} .
$$

In case $p_{j}>q_{j}$ a circular sum can be calculated by

$$
S_{j}=S\left(p_{j}, q_{j}\right)=\sum_{i=1}^{q_{j}} k_{i}+\sum_{i=p_{j}}^{n} k_{i}
$$

Easy to see that the maximum number of distinct circular sums $S_{n}$ of the ring sequence is

$$
S_{n}=n(n-1)+1 \text {. }
$$

An $n$-element ring sequence $K_{n}=\left\{k_{1}, k_{2}, \ldots\right.$, $\left.k_{i}, \ldots k_{n}\right\}$ of natural numbers for which the set of all $S_{n}$ circular sums consists of the numbers from 1 to $S_{n}=n(n-1)+1$, that is each number occurring exactly once, let us define a "golden numerical ring".

Comparing Eqs. (1) and (4), we see that the number of sums $S_{\max }^{\prime}$ for consecutive terms in the ring topology is nearly double the number of sums $S_{n}$ in the daisy-chain topology, for the same sequence of $n$ terms.

Here is an example of GNR $\{1,3,2,7\}$ with parameters $n=4, S_{n}=13$ (Fig. 2).

Table of sums of consecutive terms in ring sequence $K_{n}=\{1,3,2,7\}$ is below (Table II). A sum of consecutive terms in the ring sequence can have any of the $n=4$ terms as its starting point $p_{j}$, and finishing point $q_{j}$, and can be of any length (number of terms) from 1 to $n-1=3$.

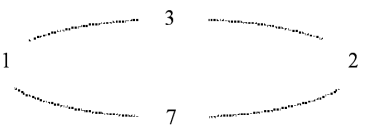

Fig. 2. The GNR $\{1,3,2,7\}$ with parameters $n=4$, $S_{n}=13$.

TABLE II

Sums of consecutive terms in the GNR $\{1,3,2,7\}$.

\begin{tabular}{c|c|c|c|c}
\hline \hline \multirow{2}{*}{$p_{j}$} & \multicolumn{4}{|c}{$q_{j}$} \\
\cline { 2 - 5 } & 1 & 2 & 3 & 4 \\
\hline 1 & 1 & 4 & 6 & - \\
2 & - & 3 & 5 & 12 \\
3 & 10 & - & 2 & 9 \\
4 & 8 & 11 & - & 7
\end{tabular}

To see this, we observe

$$
\begin{array}{lll}
1=1 & 5=3+2 & 9=2+7 \\
2=2 & 6=1+3+2 & 10=2+7+1 \\
3=3 & 7=7 & 11=7+1+3 \\
4=1+3 & 8=7+1 & 12=3+2+7
\end{array}
$$

So, for the ring sequence $\{1,3,2,7\}$ the set of all $S_{n}=$ 12 circular sums consists of numbers from 1 to 12 , where each number occurs exactly once $(R=1)$.

\subsection{More general type of golden numerical ring}

Next, we consider a more general type of GNR, where the $S=S_{n}-1$ circular sums of consecutive terms give 
us each integer value from 1 to $S$, for some integer $S$, exactly $R$ times, as well as the sum of all $n$ terms exactly once. Here we see that

$$
S=n(n-1) / R \text {. }
$$

There is example of GNR $\{1,1,2,3\}$, where $n=4$ and $R=2$, so that $S=6$.

To see this, we observe

$$
\begin{array}{lll}
1=1 & 3=3 & 5=2+3 \\
1=1 & 3=1+2 & 5=3+1+1 \\
2=2 & 4=3+1 & 6=1+2+3 \\
2=1+1 & 4=1+1+2 & 6=2+3+1
\end{array}
$$

We see that each circular sum from 1 to 6 occurs exactly twice $(R=2)$.

\subsection{Two-dimensional golden numerical rings}

Let us regard the $n$-element ring sequence $K_{2 \mathrm{D}}=$ $\left\{\left(k_{11}, k_{12}\right),\left(k_{21}, k_{22}\right), \ldots\left(k_{i 1}, k_{i 2}\right) \ldots,\left(k_{n 1}, k_{n 2}\right)\right\}$, where we require all terms in each modular vector-sum to be consecutive 2-element sequences as elements of the sequence. A modular vector-sum of consecutive terms in the ring sequence can have any of the $n$ terms as its starting point, and can be of any length from 1 to $n-1$. An $n$-stage ring sequence $K_{2 \mathrm{D}}=\left\{\left(k_{11}, k_{12}\right),\left(k_{21}, k_{22}\right)\right.$, $\left.\ldots\left(k_{i 1}, k_{i 2}\right), \ldots\left(k_{n 1}, k_{n 2}\right)\right\}$, for which the set of all

$$
S_{2 \mathrm{D}}=S=n(n-1) / R
$$

modular vector-sums forms two-dimensional grid, where each node of the grid occurs exactly $R$ times, we call two-dimensional golden numerical ring (2D GNR).

Next, we consider two-dimensional $n$-element ring sequence GNR with four $(n=4)$ terms in the ring topology, where $k_{1}=(0,2), k_{2}=(1,0), k_{3}=(1,1), k_{4}=(2,2)$.

We can calculate easily all the two-dimensional modular vector-sums, taking modulo $m_{1}=3$ for the first component of vector-sum and modulo $m_{2}=4$ for its second component.

Here we see

$$
\begin{array}{llll}
(0,1) \equiv(1,1)+(2,2)+(0,2) ; & (0,3) \equiv(1,1)+(2,2) ; & & (1,2) \equiv(0,2)+(1,0) ; \\
(1,3) \equiv(1,0)+(1,1)+(2,2) ; & (2,0) \equiv(2,2)+(0,2) ; & & (2,1) \equiv(1,0)+(1,1) ; \\
(2,3) \equiv(0,2)+(1,0)+(1,1) ; & (0,0) \equiv(2,2)+(0,2)+(1,0) . & &
\end{array}
$$

So long as the elements $(0,2),(1,0),(1,1),(2,2)$ of the ring sequence themselves are vector-sums too, the set of modular vector-sums configures $3 \times 4$ matrix as follows:

$$
\begin{array}{llll}
(0,0) & (0,1) & (0,2) & (0,3) \\
(1,0) & (1,1) & (1,2) & (1,3) \\
(2,0) & (2,1) & (2,2) & (2,3)
\end{array}
$$

The result of the calculation forms the $3 \times 4$ grid which exhausts the 2D modular vector-sums and each of it occurs exactly once $(R=1)$. So, the ring sequence of the $2 \mathrm{D}$ vectors $\{(0,2),(1,0),(1,1),(2,2)\}$ is two-dimensional golden numerical ring (2D GNR) with $n=4, R=1$, and $m_{1}=3, m_{2}=4$.

\section{Construction of non-redundant arrays based on GNRs}

\subsection{Linear non-redundant arrays}

To avoid interference of the components of the same spatial frequency which may lead to ambiguity, the arrays should be non-redundant $[2,3]$. The method for constructing linear non-redundant array of $n$ elements, based on the Golomb ideal ruler with marks $0,1,4,6$ is illustrated below (Fig. 3). Easy to see we use $n$-element daisy-chain sequence of distinct positive integers $\{1,3,2\}$, which for all sums of consecutive terms allows an enumeration of all numbers $1,2, \ldots, 6=1+3+2$.

Here is a non-redundant array, based on the Golomb ideal ruler with marks 0, 1, 4, 6 (Fig. 3).

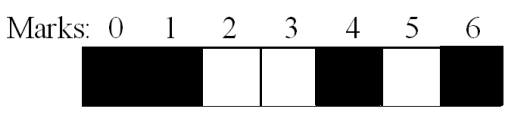

Fig. 3. A linear non-redundant array, based on the Golomb ideal ruler with marks 0, 1, 4, 6 .

Unfortunately, there is no Golomb ideal rulers for $n>3$. In this connection there is a need for developing methods of non-redundant arrays constructing when the arrays are large. The problem is especially pressing for constructing $n$-element non-redundant arrays over $m_{1} \times m_{2}$ grids for an arbitrary $n[2]$.

\subsection{Planar non-redundant arrays}

The method for constructing non-redundant two-dimensional (planar) $n$-element arrays over $m_{1} \times m_{2}$ grids, or under sizes, is suggested in the next example, where

$$
m_{1} m_{2}=1+n(n+1) / R .
$$

The method is based on the use of $2 \mathrm{D} \operatorname{GNR}\{(0,0)$, $(0,2),(0,4),(1,1),(1,3),(2,1),(2,2),(2,3),(3,2),(4,2)$, $(5,0),(5,4)\}$ for parameters $n=13, R=4, m_{1}=5$, $m_{2}=8$, where $m_{1} \times m_{2}=5 \times 8=40$. Figure 4 presents a planar non-redundant array on the $5 \times 6$ matrix reconstructed from the $5 \times 8$ grid by crossing out two columns.

We search needed solution after construction of 2D matrix of all circular two-dimensional modular vector-sums 


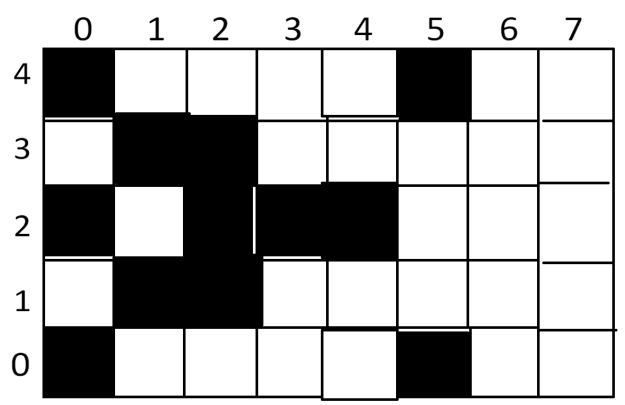

Fig. 4. Non-redundant array over $5 \times 6$ grid reconstructed from the over $5 \times 8$ grid based on the use of $2 \mathrm{D}$ GNR $\{(0,0),(0,2),(0,4),(1,1),(1,3),(2,1),(2,2)$, $(2,3),(3,2),(4,2),(5,0),(5,4)\}$.

on the golden numerical ring and regarding each of it with respect to search minimum of the sum using crossing out. Underlying procedures make it possible to configure planar non-redundant arrays with the smallest possible number of grids.

The example shows even more - that the grid order can be reduced further with loss of the possibility to construct an array.

\subsection{D non-redundant arrays}

The method for constructing non-redundant three-dimensional $n$-element arrays over $m_{1} \times m_{2} \times m_{3}$ grids, or under sizes, is suggested in the next example. The three-dimensional $(t=3)$ GNR of order $n$ can be represented as $n$-element ring sequence $\left\{\left(k_{11}, k_{21}, k_{31}\right),\left(k_{12}, k_{22}, k_{32}\right)\right.$, $\left.\ldots,\left(k_{1 n}, k_{2 n}, k_{3 n}\right)\right\}$, where all modular $3 \mathrm{D}$ vector-sums of consecutive terms in the ring sequence of the $n$ terms occurs exactly $R$ times. Let the first of six $(n=6)$ mask elements is the $(0,0,0)$ cell of $2 \times 3 \times 5-$ matrix cycling. Now, we can obtain coordinates of the remaining five elements accordingly the underlying $3 \mathrm{D}$ perfect distribution cycling modulus $m_{1}=2, m_{2}=3, m_{3}=5:\{(1,1,1)$, $(0,2,3),(1,2,1),(1,1,3),(1,2,2)\}$. We can observe the $3 \mathrm{D}$ combinatorial construction for configure 3D acoustic system of fine resolution.

\section{Conclusion}

GNRs are perfect combinatorial models of non-redundant one-, 2D or 3D space-tapered arrays of acoustic systems. These models provide their optimal structure from the point of the convenience to reproduce the maximum number of combinatorial varieties in the system with the limited number of elements. Method allows finding optimal solution in the simplest way using selected matrix of circular two- or three-dimensional modular vector-sums on the GNR as well as crossing out operations. These procedures make it possible to configure high performance arrays of acoustic systems with respect to resolving ability, including synthesis of wide-aperture equipped with non-redundant sets of opening for space and underwater acoustics. There exists a priori an infinite set of GNRs, and their parameters can be of any large number.

\section{References}

[1] M. Hall Jr., Combinatorial Theory, Wiley, New York 1986.

[2] S.W. Golomb, in: Surveys in Combinatorics - 1991, Ed. A.D. Keedwell, Cambridge University Press, London 1991, p. 1.

[3] L.E. Kopilowich, Opt. Commun. 68, 7 (1988).

[4] V. Riznyk, in: Multidimensional Systems: Problems and Solutions, IEE, Savoy Place, London 1998, p. 5/1. 